\title{
Peridynamic model for nonlinear viscoelastic creep and creep rupture of Polypropylene
}

\begin{abstract}
This paper presents the peridynamic numerical method for nonlinear viscoelastic creep behaviour which consists of primary, secondary, tertiary creep stages and creep rupture. A nonlinear viscoelastic creep constitutive equation based on internal state variable (ISV) theory which covers four creep stages is examined. The viscoelastic equation is substituted into material parameter in the peridynamic equation to derive a new peridynamic method with two time parameters i.e. numerical time and real time. The parameters of the viscoelastic equation is analyzed and evaluated. In validating this peridynamic method, a comparison is made between numerical and experimental data. The peridynamic method for nonlinear viscoelastic creep behaviour (VE-PD) is approved by the good similarity between numerical and experimental creep strain curves with overall difference of $10.67 \%$. The nonlinearity of experimental and numerical data is adequately similar as the error between experimental and numerical curves of secondary stage strain rate against load is $8.022 \%$. The shapes of fractured numerical specimen show good resemblance with the experimental result as well.
\end{abstract}

Keyword: Peridynamic; Viscoelastic; Creep; Polypropylene; Nonlinear; Fracture 